\title{
Metas académicas, atribuciones causales y género: su determinación en el rendimiento académico del alumnado de bachillerato
}

\author{
Academic goals, causal attributions and gender: their assessment in
}

\author{
high school students' academic performance
}

\author{
Indalecio Ramudo Andión, Alfonso Barca Lozano, Juan Carlos Brenlla Blanco, Eduardo Barca Enríquez \\ Universidade da Coruña.
}

\begin{abstract}
Resumen
Este trabajo trata de analizar la influencia de variables motivacionales como Metas académicas y Atribuciones causales sobre el rendimiento del alumnado de bachillerado, analizado diferencialmente por sexo y especialidad. Para ello disponemos de una muestra de 1505 sujetos, pertenecientes a las tres especialidades que comprende la Enseñanza Media de Galicia. Mediante análisis correlacionales y de regresión lineal múltiple se obtienen resultados que demuestran una relación positiva y significativa de las atribuciones internas sobre el rendimiento, resultando mayor esta relación en el caso de las mujeres. Respecto a las Metas de aprendizaje se aprecia la misma influencia positiva sobre el rendimiento, siendo ésta mayor respecto a los hombres. Finalmente, se proponen actividades específicas de apoyo motivacional con alumnado de bajo rendimiento.

Palabras clave: Metas académicas; metas de aprendizaje, metas de rendimiento, metas de valoración social, atribuciones causales internas y externas, atribucuiones causales incontrolables y falta de esfuerzo.
\end{abstract}

\begin{abstract}
This survey tries to analyze the influence of changeable motivationals such as Academic Goals and Causal Attributions on High School students' performance, through sex and specialty analyses. To achieve this, we have a sample of 1505 subjects, belonging to three common areas in Galician High School. The correlational and multiple linear regression analyzes performed show a positive and significant relationship between the internal performance attributions, being this relationship greater for women. Regarding learning goals, the same positive performance influence is observed, which is more considerable in relation to men. Finally, specific motivational support activities are proposed to low performance students.

Keywords: Academic goals; Learning goals, performance goals, social assessment goals, internal and external causal attributions, uncontrollable causal attributions and lack of effort.
\end{abstract}

\section{Las metas académicas}

Las metas académicas, son concebidas por distintos autores (Pintrich et als 2006), como el motivo que mueve al individuo a realizar una tarea.
La mayoría de los autores que trabajan sobre metas se sitúan en la perspectiva de las múltiples metas, lo cual significa que los alumnos no tienen siempre la misma motivación, es decir, no emplean un único tipo de meta, sino que optan por distintas metas en función de sus intereses personales hacia la actividad a desarrollar y del contexto en que se desenvuelva. En función de estas circunstancias el individuo opta por metas de: aprendizaje (relacionadas on la comprensión, dominio) rendimiento (vinculadas con las buenas calificaciones) y/o valoración social (relacionadas con la necesidad de ser valorados positivamente en su entorno.

\section{Metas académicas y diferencias de género}

Las diferencias de género en relación con la motivación de logro fueron estudiadas, entre otros autores, por Barberá (1998), llegando a la conclusión de que el logro, para los hombres, siempre incluye

instrumentalidad y reconocimiento público, mientras que las mujeres valoran de manera intrínseca las cosas bien hechas. En esta misma línea, Anderman y Anderman, (1999) demostraron que los hombres tienen una motivación máis extrínseca que las mujeres.

\section{Relación de las metas con el rendimiento académico}

De los distintos estudios realizados se deduce la existencia de una cierta relación entre determinado tipo de metas y el rendimiento académico, encontrándose una clara correlación positiva entre las metas de aprendizaje y el nivel de competencia adquirido, así como entre las metas de rendimiento/logro y los resultados académicos, mientras que, por el contrario, las metas de valoración social correlacionan negativamente con el rendimiento (Barca, Peralbo, Porto, Malmierca y Brenlla, 2011); sin embargo, tal y como demostraron distintos autores, como, Harackiewicz, Barron, Tauer, Carter y Elliot (2000) el alumnado que consigue mejores resultados, tanto a nivel de rendimiento como de competencia en una materia determinada, son los que adoptan los dos tipos de metas, de aprendizaje y de logro. Esta idea nos lleva a la conclusión de que lo mas adaptativo y lo que hacen la mayoría de alumnos/as, es optar por múltiples metas, 
eligiendo unas $\mathrm{u}$ otras en función de las variables personales o contextuales presentes en el momento.

\section{Las atribuciones causales}

Las atribuciones causales se consideran el determinante primario de la motivación y se entienden como la manera en que las personas explican las razones o causas de sus éxitos o fracasos. Por lo tanto, después de una situación de aprendizaje ante la que se produzca un resultado inesperado el alumno se pregunta sobre las causas que pudieron provocar dicho resultado, las cuales pueden ser muy variadas, aunque la mayoría de los autores coinciden en hablar de cuatro causas fundamentales: capacidad, dificultad de la tarea, esfuerzo, suerte o azar. Causas que a su vez se engloban en una serie de categorías o dimensiones que ayudan a delimitarlas: interna/externa, estable/inestable y controlable/incontrolable.

\section{Diferencias de género en las atribuciones causales}

Numerosos estudios realizados con estudiantes de secundaria encuentran una clara diferenciación entre alumnos y alumnas en cuanto a sus atribuciones causales, tal y como reconocen, entre otros autores, Durán-Aponte y Pujol, (2012) o, Inglés, Díaz, García y Ruiz (2011). Mientras los alumnos atribuyen sus buenos resultados académicos a la capacidad, ellas, lo hacen al esfuerzo (Almeida et al. 2008). Además, los chicos atribuyen significativamente más que las chicas sus fracasos a causas externas e inestables, con lo que tienden a preservar su autoconcepto y autoestima (Covington, 2000). De todas formas, es importante tener en cuenta que esta diferenciación entre hombres y mujeres en cuanto a las atribuciones que realizan de sus éxitos y fracasos está muy relacionada con la materia sobre la que se pregunte (científica o humanista), lo cual se demuestra en diferentes investigaciones realizadas sobre el tema (Inglés, Díaz, García y Ruíz, 2011).

\section{Atribuciones causales y rendimiento académico}

El rendimiento académico de los estudiantes, además de estar influído por otros muchos factores, se encuentra enormemente afectado por la adopción de unos u otros patrones atribucionales (Fernández de Mejía, Arnáiz, Mejía, Barca, 2015; Ramudo, 2015). De esta forma, tal y como descubrieron Morales y Gómez, (2009), la motivación estará favorecida en la medida en que las atribuciones pertenezcan a la dimensión interna, donde se atribuye a uno mismo la responsabilidad del éxito y del fracaso, especialmente al esfuerzo (Valle, Rodríguez y Regueiro, 2015); a la dimensión inestable, donde aquello que causa el éxito o fracaso puede ser modificado; a la dimensión controlable, porque el sujeto concibe situaciones controlables por él mismo. Pues bien, con este trabajo se trata de analizar las relaciones e influencia de variables motivacionales, como las metas académicas y atribuciones causales, en función del género, sobre el rendimiento académico del alumnado de bachillerato.

\section{Método}

\section{Participantes}

Los sujetos de la muestra $(\mathrm{N}=1.505)$ proceden mayoritariamente de las provincias de A Coruña y Pontevedra distribuídos en 10 Institutos de Enseñanza Secundaria en A Coruña y 12 en Pontevedra. De Ourense proceden de 4 Institutos y de Lugo de 5. La distribución por provincias se corresponde, en buena medida, con la real de la población de alumnado de bachillerato en las cuatro provincias de Galicia.

En cuanto a la distribución por sexos, se aprecia una ligera predominancia de sujetos de sexo femenino (el $58.7 \%$ de la muestra), frente a los de sexo masculino que constituyen el $41.3 \%$ restante, lo cual concuerda en buena medida con el reparto por género de la población real. Por cursos se distribuye el $59.1 \%$ en alumnado de $1^{\circ}$ curso y el $40.9 \%$ restante se corresponde con el de $2^{\circ}$ curso.

\section{Instrumentos}

1. Cuestionario de Datos Personales, Familiares y Académicos (CDPFA-57), que cuenta con los siguientes apartados: Datos personales y académicos del alumno/a; rendimiento académico; concepción del fracaso escolar; lugar y tiempo de dedicación al estudio en casa; metas académicas....me esfuerzo en mis estudios porque...

2. Escala de Valoración de la Autoeficacia (EVA) que cuenta con los siguientes apartados: autoeficacia general; creencias de autoeficacia académica; autoeficacia y tareas de estudio.

3. Sistema integrado de evaluación de atribuciones causales y procesos de aprendizaje para el alumnado de educación secundaria (SIACEPA), en concreto la subescala EACM: Subescala de atribuciones causales.

El Rendimiento académico se ha cuantificado para cada alumno a partir de sus expedientes de calificaciones académicas correspondientes al curso anterior y las evaluaciones obtenidas hasta la fecha de administración de las pruebas.

\section{Procedimiento}

Todos los datos se han obtenido directamente en los propios centros de esneñanza, en las aulas ordinarias, una vez que se había obtenido la autorización pertinente de la dirección. Se administraron los instrumentos de evaluación adaptados a los estudiantes en una única sesión después de insistirles que su participación era voluntaria y totalmente anónima sin repercusión alguna en sus calificaciones académicas. 


\section{Técnicas de análisis}

Se utilizan medidas de tendencia central y un análisis de regresión lineal múltiple con el objeto de analizar la influencia de variables de metas académicas y atribuciones causales sobre el rendimiento académico y en función del género.

\section{Resultados}

Se ha realizado un análisis de regresión lineal múltiple mediante el cual se trataba de comprobar, diferencialmente por sexos, la capacidad predictiva de las variables motivacionales relacionadas con las atribuciones causales y metas académicas sobre el rendimiento académico del alumnado de bachillerato. Se trata de comprobar si las atribuciones causales y las metas académicas tienen una influencia importante sobre el rendimiento académico del alumnado, y si existen diferencias por sexos en cuanto a esta tendencia.

El modelo de regresión resulta estadísticamente significativo tanto para las mujeres $\left(\mathrm{F}_{(6,883)}=40.085\right.$, $\mathrm{p}<.01)$ como para los hombres $\left(\mathrm{F}_{(3,620)}=42.930, \mathrm{p}<\right.$ $.01)$.

Lo primero que se aprecia es que las variables atribucionales y metas académicas explican, en el caso de las alumnas de la muestra el $21.1 \%$ de la varianza total explicada del rendimiento, y en los alumnos el $17,3 \%$ (ver tabla 1 ).

Estos datos muestran que el porcentaje de varianza total explicada del rendimiento, de las variables motivacionales estudiadas (atribuciones causales y metas académicas) es moderadamente buena, pero no homogénea en los dos grupos, apreciándose importantes diferencias, las cuales se manifiestan en un mayor porcentaje de varianza explicada en las mujeres.
De esta manera se comprueba cómo, en las alumnas, las atribuciones que mejor predicen el rendimiento son las atribuciones internas, atribuciones incontrolables/falta de esfuerzo y baja capacidad. En relación con las metas académicas, nos encontramos con los tres tipos: metas de aprendizaje, metas de rendimiento/logro y metas de rendimiento/valoración social. En el caso de los hombres, las variables motivacionales que mejor pronostican el rendimiento según los resultados obtenidos son las metas de aprendizaje; en cuanto a las atribuciones, estarían las incontrolables/falta de esfuerzo e internas. Destacaría siempre la primera variable de la ecuación como la que mayor poder explicativo tiene sobre la varianza total; en este caso serán, en las mujeres, las atribuciones internas y en los hombres las metas de aprendizaje.

En relación con las alumnas-mujeres, el factor atribución interna es el que tiene un mayor peso, explicando el $9.3 \%$ de la varianza total, siendo su beta positiva $(\beta=.304)$, lo cual nos indica que la relación con el rendimiento académico es muy direccionalmente positivo. Esto quiere decir que las mujeres que imputan su buen rendimiento a causas internas, como buena capacidad, inteligencia, habilidades personales, etc.; éstas actúan como agentes motivadores del aprendizaje $\mathrm{y}$ del buen rendimiento.

Otro factor que también tiene un peso importante es el factor atribuciones incontrolables/escaso esfuerzo, con una explicación de la varianza del $7.3 \%$ y con beta negativa $(\beta=-.271)$, lo cual indica que las mujeres que atribuyen su rendimiento a variables sobre las que carecen de todo tipo de dominio o control o falta de esfuerzo obtienen un rendimiento académico negativo, bajo (ver tabla 1).

Tabla 1

Modelo predictivo de Regresión Lineal Múltiple de Atribuciones Causales y Metas Académicas sobre el Rendimiento Académico.

\begin{tabular}{|c|c|c|c|c|c|c|c|c|c|}
\hline & & $n=1505$ & $\mathbf{R}^{2} \mathbf{N}$ & ujeres & $21.5 \% \mathbf{R}^{2}$ & mbres $=17,3 \%$ & & & \\
\hline & Pasos & Variables & $\mathbf{R}$ & $\mathbf{R}^{2}$ & $\begin{array}{c}\mathbf{R}^{2} \\
\text { corrixida } \\
\end{array}$ & $\begin{array}{c}\text { Error tip. de } \\
\text { estimación }\end{array}$ & $\begin{array}{c}\text { Cambio } \\
\text { en } \mathbf{R}^{2}\end{array}$ & $\beta$ & Sig. \\
\hline \multirow{6}{*}{$\begin{array}{c}\text { Femenino } \\
(\mathrm{n}=884)\end{array}$} & 1 & $A T B / I N T$ & .304 & .093 & .092 & .742 & .093 & .304 & .000 \\
\hline & 2 & $A T B / I N C-F E$ & .407 & .166 & .164 & .712 & .073 & -.271 & .000 \\
\hline & 3 & $M E / A P$ & .434 & .189 & .186 & .702 & .023 & .159 & .000 \\
\hline & 4 & $M E / V S$ & .454 & .206 & .202 & .695 & .017 & -.137 & .000 \\
\hline & 5 & $M E / R E$ & .460 & .211 & .207 & .693 & .005 & .081 & .014 \\
\hline & 6 & $A T B / B C P$ & .464 & .215 & .210 & .692 & .004 & -.065 & .035 \\
\hline \multirow{3}{*}{$\begin{array}{l}\text { Masculino } \\
\qquad(\mathrm{n}=621)\end{array}$} & 1 & ME/AP & .300 & .090 & .089 & .724 & .090 & .300 & .000 \\
\hline & 2 & $A T B / I N C-F E$ & .365 & .133 & .130 & .707 & .043 &.-210 & .000 \\
\hline & 3 & $A T B / I N T$ & .416 & .173 & .169 & .692 & .039 & .208 & .000 \\
\hline
\end{tabular}

Resultados del Análisis de Regresión, método de pasos sucesivos. Variables independentes de la Escala EACM: Atribuciones Causales Internas (ATB/INT), Atribuciones Causasles Externas (ATB/EXT), Atribuciones Causales Incontrolables o/y Escaso Esfuerzo (ATB/INC-EE), Atribuciones Causales a la Baja Capacidad; Metas Académicas: Metas de Aprendizaje (ME/AP) Metas de Logro (ME/RE) y Metas de Valoración Social (ME/VS)Variable dependente: Rendimiento Académico. 
La contribución a la ecuación de regresión de las metas de aprendizaje es más moderada, con una explicación de la varianza del $2.3 \%$ y con una beta positiva $(\beta=.159)$, lo que significa que aquellas alumnas que aprenden de una manera significativa, construyendo nuevos conocimientos a partir de los ya existentes y que ven el aprendizaje como una oportunidad de formación y disfrute serán sujetos altamente motivados y con un buen rendimiento académico.

Las metas de rendimiento ligadas a la valoración social mantienen un peso explicativo de la varianza similar a las anteriores, siendo de un $1.7 \%$, aunque la beta, a diferencia de las metas de aprendizaje es negativa $(\beta=-137)$ lo que significa que las mujeres que realicen sus tareas para obtener halagos, recompensas, evitar ser rechazadas, etc., obtendrán, generalmente, bajas calificaciones (ver tabla 1).

En un lugar considerablemente más alejado de las anteriores en cuanto a la contribución a la explicación de la varianza se refiere, están las metas de logro, que explican solamente un $0.5 \%$ de la varianza total y con una beta positiva $(\beta=.081)$, lo que indica una relación positiva con el rendimiento. También se incluiría en este grupo de variables con un bajo porcentaje de la varianza total, las atribuciones a la baja capacidad, que explican un $0,4 \%$ de dicha varianza, aunque aquí la beta es negativa $(\beta=-.065)$, lo cual significa que las alumnas que atribuyen su rendimiento a su falta de habilidades, capacidad o inteligencia obtienen rendimientos bajos (ver tabla 1).

Respecto a los alumnos, el factor metas de aprendizaje es el que tiene un mayor peso, explicando el $9 \%$ de la varianza total, con beta positiva $(\beta=.300)$, lo que nos indica que la relación de este tipo de metas con la motivación de cara al aprendizaje y al rendimiento académico es muy positiva. Por lo tanto, los alumnos que tratan de aprender para adquir más, pero sobre todo mejores conocimientos, para tener más dominio de la materia objeto de estudio; serán sujetos que disfruten con los aprendizajes con rendimientos muy positivos.

Con una contribución considerablemente más moderada se encuentran las atribuciones incontrolables/escaso esfuerzo y las atribuciones internas, con una explicación de la varianza del 4,3\% ( $\beta$ $=-.210) \mathrm{y}$ del $3.9 \%(\beta=.208)$, respectivamente, siendo su relación positiva con el rendimiento en el segundo caso, cuando se atribuye éste a causas internas, y negativo cuando se atribuye el bajo rendimiento a variables incontrolables y escaso esfuerzo (ver tabla 1).

\section{Discusión e implicaciones educativas}

Tanto en el caso de los hombres como en el de las mujeres, encontramos que las atribuciones causales internas son variables muy importantes en la explicación del rendimiento académico del alumnado de Bachillerato, aunque su importancia es considerablemente más elevada en el caso de las mujeres, llegando a duplicar en porcentaje los casos explicados respecto a los de sus compañeros $(9.3 \%$ frente a $3.9 \%$ ). En ambos casos es positiva a su relación con el rendimiento (en mujeres, $\beta=304$; en hombres, $\beta$ = 208), aunque también sensiblemente superior en el caso de las mujeres. Esto significa que, en relación con la adopción de atribuciones causales internas por parte del alumnado de Bachillerato, es manifestamente mayor el porcentaje de alumnas-mujeres que adoptan este tipo de atribuciones (que atribuyen sus resultados a variables intrínsecas como la capacidad, habilidad, inteligencia, etc.) respecto a los hombres, siendo también superior su rendimiento académico en relación a éstos, y que también realizan este tipo de atribuciones.

Las metas de aprendizaje también destacan por su capacidad predictiva sobre el rendimiento académico, sobre todo en el caso de los hombres, ya que, a diferencia de lo que pasaba con las atribuciones causales, ahora el porcentaje de hombres que adopta este tipo de metas es muy superior al de mujeres llegando incluso a triplicarlo $(9 \%$ de hombres, frente a $2.3 \%$ de mujeres). Las betas son positivas en ambos casos (hombres, $\beta=.300$ e mujeres, $\beta=.159$ ), como se puede observar también mucho más elevada en el caso de los hombres. Por esto, se puede decir que las metas de aprendizaje, a pesar de que son empleadas por la mayoría del alumnado de Bachillerato, son adoptadas en mayor medida por los hombres frente a las mujeres; además, entre los que optan por este tipo de metas, son los varones los que obtienen calificaciones más elevadas.

Respecto a las metas de rendimiento/logro y rendimiento/valoración social, se afirma que su presencia es mucho más discreta, ya que en el caso de los hombres no llegan ni a incluírse en el modelo. En el caso de las mujeres representan un porcentaje bastante discreto de la varianza total explicada; por lo que su importancia es más bien escasa, según los resultados que se aprecian en la tabla 1.

En cuanto a las implicaciones educativas hay que decir que son, al menos, tres las sugerencias que nos permiten establecer los resultados obtenidos en este trabajo:

1) Necesidad de potenciar actividades de enseñanza significativas

Se trata de aprovechar la óptima potenciación que se da tanto en los alumnos como en las alumnas, de las metas de aprendizaje y de su valoración del esfuerzo y la capacidad para deesarrollar tareas y actividades de aprendizaje. Las atribuciones causales internas y las metas de aprendizaje son importantes para este cometido de aprovechamiento de las potencialidades del alumnado, tanto para la organización de contenidos, secuenciación por gradiente de dificultad como para su comprensión y su relación con otros contenidos próximos e implicativos. El profesorado debería aprovechar la óptima disponibilidad que el alumnado presenta en estas lineas señaladas de metas y atribuciones. Es relevante la diferencia que existe entre mujeres y hombres en la adopción de atribuciones internas, por eso deberá centrarse el profesorado quizás más en los alumnos, quienes, por sus escasas creencias de que son capaces de realizar con éxito las tareas de aprendizaje es posible con su esfuerzo y su capacidad, es decir, deben convencerse de que todo será posible si 
le dedican tiempo, concentración, buscando información adecuadamente y resumiendo o haciendo esquemas que les faciliten la retención y comprensión de los contenidos. La consigna será: hacérselo ver, hacérselo saber y hacérselo creer.

2) Atención al alumnado con bajo nivel de aprendizaje y rendimiento

Es importante destacar este hecho, ya que existe un número de alumnos y alumnas con bajo nivel de implicación en los contenidos a aprender y comprender. Es preciso implicarles con actividades que despierten sus intereses, tanto desde su atracción visual, como de atracción física y mental, diseñando actividades para clase que puedan tener este tipo de características y, a su vez, que puedan realizarlas con éxito y potenciando y valorando sus esfuerzos en la ejecución de las mismas.

3) Potenciar la motivación intrínseca

Por una parte, destacar la necesidad de que la implicación en las actividades de aula y para casa, sobre todo en las materias algo más complejas, deberá salir siempre desde un interés personal de cada alumno. Por otra parte, para ello se debe prestar atención por parte del profesorado a este hecho de implicación, fomentando y valorando su adopción e informando a las familias de la necesidad de este tipo de implicación personal. Finalmente, es preciso que se tome conciencia de los beneficios que supone para el alumnado el hecho de hacerse responsables y protagonistas de sus propios aprendizajes y, en consecuencia, de su rendimiento.

\section{Referencias}

Alonso, J. (2005). Motivar en la escuela, motivar en la familia.Madrid: Morata.

Anderman, L.H. y Anderman, E.M. (1999). Social predictors of changes in students achievement goal orientations. Contemporary Educational Psychology, 25, 21-37.

Barberá, E.(1998). Psicología del género. Madrid: Ariel.

Barca, A., Peralbo, M., Porto, A., Malmierca, J.L. y Brenlla, J.C. (2011). Metas académicas del alumnado de educación secundaria obligatoria (ESO) y Bachillerato con alto y bajo rendimiento escolar. Revista de educación. 354, 341-368.

Covington, M. V. (2000). Goal theory, motivation and school achievement: An integrative review. Annual Review of Psychology, 51, 171-200.

Durán-Aponte E., Pujol, L. (2012). Diferencias de género y área de estudio en las atribuciones causales de estudiantes universitarios. Anales Universidad Metropolitana, 12, (2), 39-51.

Eccles, J. S. y Midgley, C. (1989). Stage-environment fit: developmentally appropriate classrooms for young adolescents. En, Ames, C.e Ames, R. (Eds.). Research on Motivation in Education. (pp.139-186). San Diego, California: Academic Press.

Fernández de Mejía (2010). Motivación, aprendizaje y rendimiento académico en estudiantes de primer año de universidad en República Dominicana. Murcia: Universidad de Murcia (Tesis doctora inédita).
Fernández de Mejía, A., Arnáiz, P., Mejía, R. \& Barca, A. (2015). Atribuciones causales del alumnado universitario de República Dominicana con alto y bajo rendimiento académico. Revista de estudios e investigación en psicología y educación. ISSN: e2386-7418. Vol. 2 (1), 19-29. DOI: 10.17979/reipe.2015.2.1.1319.

Harackiewicz, J.M., Barron, K.E., Tauer, J.M., Carter, S.M. y Elliot, A.J. (2000). Short- term and long-term consequences of achievement goals: Predicting interest and performance over time. Journal of Educational Psychology, 92, 316- 330.

Inglés, C.J., Díaz, Á., García, J. M. y Ruiz C. (2011). El género y el curso académico como predictores de las atribuciones en lectura y matemáticas en estudiantes de Educación Secundaria Obligatoria. Anales de Psicología, 27 (2), 381-388.

Morales, P. y Gómez, V. (2009). Adaptación de la escala atribucional de motivación de logro de Manassero y Vázquez. Investigación Pedagógica, 12, (3), 33-52.

Pintrich, P.R. y Schunk, D.H. (2006). Motivación en contextos educativos. Teoría, investigación y aplicaciones ( $2^{\mathrm{a}}$ ed.). Madrid: Pearson Educación.

Ramudo Andión, I. (2015). Variables motivacionais, autoeficacia e enfoques de aprendizaxe en relación co rendemento académico do alumnado de bacharelato. Universidade da Coruña (Tesis doctoral inédita).

Valle, A., Rodríguez, S. y Regueiro, B. (2015). Motivación y aprendizaje escolar. CCS: Madrid. 